\title{
A note on implicit premisses
}

\section{David Hrtchcock McMaster University}

In a recent article in this journal, Geoffrey Goddu (2002, p. 15) asserts in passing that in the argument:

(1) All monkeys are primates, so with certainty all monkeys are mammals, the weakest implicit premiss that will make the argument valid is

(2) All primates are mammals.

This assertion is false; the following weaker premiss will make (1) valid:

(3) Either not all monkeys are primates or all monkeys are mammals.

It is clear that (3) makes (1) valid, since adding it as a premiss produces a disjunctive syllogism of the form Either not $A$ or $B ; A$; so $B$.

To prove that (3) is weaker than (2), note first that (2) entails (3). For suppose (2) is true but (3) is false. Then, by the falsehood of (3), we have: neither not all monkeys are primates nor all monkeys are mammals. That is: All monkeys are primates, but not all monkeys are mammals.

Since all monkeys are primates and also all primates are mammals (by 2), then all monkeys are mammals. But according to our supposition not all monkeys are mammals. So we have a contradiction, which means the supposition is false. QED

Note second that (3) does not entail (2). For consider the following possible situation in which (3) is true but (2) is false. The world includes two unusual animals, a monkey named Monk who is not a primate and a primate named Prim who is not a mammal. (We ignore the possible objection that it is part of the meaning of 'monkey' that all monkeys are primates and part of the meaning of 'primate" that all primates are mammals, since the claim that (1) is invalid as it stands implies that primates are not by definition mammals and by analogy we may suppose that monkeys are not by definition primates. Alternatively, one may change the example.) The existence of Monk shows that not all monkeys are primates; hence either not all monkeys are primates or all monkeys are mammals, i.e., "either not all monkeys are primates or all monkeys are mammals" is true. And the existence of Prim shows that "all primates are mammals" is false. QED

Thus the implicit premiss approach to arguments like (1) has to explain why we should propose (2) rather than (3) as implicit in (1). Any adequate explanation should have a good theoretical motivation and should generate implicit premisses in particular cases which correspond to the implicit component which skilled argument analysts intuitively supply (unless their intuitions in the given case can be shown in some non-circular way to be misguided). Although I cannot prove it in 
this brief note, to the best of my knowledge no published explanation meets these two criteria of adequacy.

An alternative approach can explain without difficulty why (2) rather than (3) is implicit in (1). Any argument involves the claim that its conclusion follows from its premiss(es). This claim is the claim that there is some general rule of inference, possibly qualified by modal qualifiers like 'probably' or 'presumably', in virtue of which the conclusion follows. And any such rule is legitimate if and only if the corresponding generalized conditional is non-trivially true (or is an instance of a generalized conditional which is non-trivially true), in the sense that its antecedent is not always false and its consequent is not always true. In (1) the modal qualifier 'with certainty' indicates that the claimed general rule of inference is exceptionless. Only one exceptionless general rule of inference which would license the step from premiss to conclusion in (1) has a chance of being legitimate, namely the rule:

(4) From a premiss of the form All As are primates, you may infer the corresponding conclusion of the form All As are mammals.

And the generalized conditional which corresponds to this rule is:

(5) For any $A$, if all $A$ s are primates, then all $A$ s are mammals.

This generalized conditional is in turn logically equivalent to the sentence,

(6) All primates are mammals.

Hence (2) is implicit in (1).

(To see that (5) is logically equivalent to (6), first suppose that (5) is true and prove (6). By instantiation on (5), if all primates are primates, then all primates are mammals. But obviously all primates are primates. Hence, by modus ponens, all primates are mammals, i.e., (6) is true. Now suppose that (6) is true and prove (5). Suppose the antecedent of (5) for some arbitrary class B: all Bs are primates. Since all primates are mammals (by 6 ), then all Bs are mammals. Hence, by conditional proof, if all Bs are primates, then all Bs are mammals. But B was arbitrarily selected, and we made no special assumptions about it. Hence in general, for any $A$, if all As are primates, then all As are mammals-i.e., (5) is true. QED)

The reader can check with other examples (e.g., any example of a one-premiss argument which can be construed as an incomplete categorical syllogism) that this pattern holds universally. The proposition that argument analysts intuitively supply is not the weakest validating premiss; rather, it is the expression of a general rule which would license the inference. The doctrine of implicit premisses is largely a myth. Theorists of argumentation and practitioners of argument analysis and evaluation should abandon it.

\section{Reference}

Goddu, G.C. (2002). The 'most important and fundamental' distinction in logic, Informal Logic 22.1:1-17. 